\title{
Attentional Focus, Dispositional Reinvestment, and Skilled Motor Performance Under Pressure
}

\author{
Robin C. Jackson ${ }^{1}$, Kelly J. Ashford ${ }^{2}$, and Glen Norsworthy ${ }^{2}$ \\ ${ }^{1}$ The University of Hong Kong, ${ }^{2}$ Brunel University
}

\begin{abstract}
Attentional processes governing skilled motor behavior were examined in two studies. In Experiment 1, field hockey players performed a dribbling task under single-task, dual-task, and skill-focused conditions under both low and high pressure situations. In Experiment 2, skilled soccer players performed a dribbling task under single-task, skill-focused, and process-goal conditions, again under low and high pressure situations. Results replicated recent findings regarding the detrimental effect of skill-focused attention and the facilitative effect of dual-task conditions on skilled performance. In addition, focusing on movement related process goals was found to adversely affect performance. Support for the predictive validity of the Reinvestment Scale was also found, with high reinvesters displaying greater susceptibility to skill failure under pressure. Results were consistent with explicit monitoring theories of choking and are further discussed in light of the conceptual distinction between explicit monitoring and reinvestment of conscious control.
\end{abstract}

Key Words: choking, explicit monitoring, goal setting

Despite having the ability to execute skills successfully, many athletes perform suboptimally in situations that magnify the importance of doing well on a particular occasion (e.g., Lewis \& Linder, 1997). The term choking under pressure has been used to describe this phenomenon, defined as "the occurrence of inferior performance despite striving and incentives for superior performance" (Baumeister $\&$ Showers, 1986, p. 361). There has been much interest in the attentional processes underpinning skilled performance and, in particular, in the role of these processes in choking. Much of this research has focused on the disruptive effect of self-focused attention on skilled activities (e.g., Baumeister, 1984; Carver \& Scheier, 1978) where self-focused attention refers to the "capacity [of the self] to take itself as the object of attention" (Fenigstein \& Carver, 1978, p. 1241).

In particular, it has been argued that increased pressure to do well heightens self-focus, resulting in conscious attention to the step-by-step processes that govern performance (Baumeister \& Showers, 1986; Beilock \& Carr, 2001; Masters, 1992).

${ }^{1}$ Institute of Human Performance, The University of Hong Kong, 111-113 Pokfulam Road, Hong Kong, SAR China; ${ }^{2}$ Dept. of xxxxxxxxxxxxx, Brunel University, Uxbridge, Middlesex, UB8 3PH, U.K. \㐱: Which dept? and please verify address 


\section{2 / Jackson, Ashford, and Norsworthy}

For example, Baumeister (1984) suggested that, under pressure, performers attempt to control their skills consciously but that "consciousness does not contain the knowledge of these skills, so that it ironically reduces the reliability and success of the performance" (pp. 610-611). Similarly, Masters used the distinctions between explicit and implicit processes, controlled and automatic processing, and declarative and procedural memory to argue that choking occurs when performers reinvest explicit knowledge or controlled processing under pressure.

\section{\IAu: Need a Main Head\}

\section{Explicit Monitoring Theories of Skill Failure}

In examining the attentional mediators of choking, Beilock and Carr (2001) used the term explicit monitoring to refer collectively to self-focus theories of choking, arguing that they ultimately refer to the allocation of attention to skill execution. Experimental evidence for explicit monitoring includes the finding that skills acquired in ways that minimize explicit knowledge about how to perform the skill are more robust under stress (Masters, 1992). By contrast, individuals who accumulate a large pool of explicit knowledge during learning remain vulnerable to performance degradation under stress (Liao \& Masters, 2002). Masters' finding was replicated by Hardy, Mullen, and Jones (1996) and has since been extended using different techniques to prevent conscious processing, including analogy learning (Liao \& Masters, 2001), other secondary tasks (Maxwell, Masters, \& Eves, 2000), and errorless learning procedures (Maxwell, Masters, Kerr, \& Weedon, 2001). Additional evidence includes the finding that self-awareness adaptation can help prevent skill failure under pressure. For example, both Lewis and Linder (1997) and Beilock and Carr (2001) found that participants who practiced a golf-putting task while being filmed subsequently performed better under pressure (induced by performance-contingent reward) than those who did not receive adaptation training.

The lack of online attention is reflected in retrospective recall of few explicit rules and relatively impoverished episodic knowledge of mechanics for task performance. For example, Beilock and Carr (2001) found that expert golfers had a large pool of generic knowledge concerning both how to assess a putt and the mechanics of setting up and executing the putting stroke, but had comparatively impoverished episodic knowledge of components of the mechanics for specific putts. The concept of "expertise-induced amnesia" (Beilock \& Carr, p. 703) might be considered expertise-induced inattention, since knowledge protocols indicated that experts had not forgotten the rules but simply attended to them less during skill execution.

Additional evidence for explicit monitoring theories comes from research on the relationship between attentional demands and expertise. For example, Beilock, Carr, MacMahon, and Starkes (2002) found that experienced golfers and soccer players using their dominant foot could perform a concurrent secondary tone- or word-monitoring task without any detrimental effect on primary motor performance. In the soccer-dribbling study, Beilock, Carr, et al. compared experienced and novice soccer players' performance on a dribbling task executed using their dominant and nondominant foot under different attention conditions. When dribbling with their dominant foot, experienced players performed fastest under dual-task conditions in 
which they had to repeat a target word that appeared once every three words. They performed slowest in the skill-focus condition, in which they were instructed to attend to and, upon hearing a tone, report which side of their foot was in contact with the ball. Consistent with the relationship between attention allocation and expertise, findings were reversed for the nondominant foot, with superior performance under skill-focused compared to dual-task conditions (see also Ford, Hodges, \& Williams, 2005; Gray, 2004).

\section{Individual Differences in the Propensity for Choking}

A related line of research has considered individual differences in the tendency to become self-focused (trait self-consciousness) and individual differences in the propensity for reinvesting conscious control (reinvestment). Baumeister (1984) argued that individuals with high trait self-consciousness would perform better under pressure than their low self-conscious counterparts because the former would have become acclimatized to performing in a self-focused state. He also hypothesized that low self-conscious individuals would outperform their high self-conscious counterparts in the absence of pressure. He found support for these predictions using a motor task that required participants to separate two rods in order to progress a ball along a slight incline. As predicted, low self-conscious participants (identified using the Self-Consciousness Scale [SCS]; Fenigstein, Scheier, \& Buss, 1975) performed better in the single-task condition, but were more adversely affected by increased pressure.

By contrast, Masters, Polman, and Hammond (1993) found a significant positive correlation between Reinvestment Scale scores and performance failure under pressure in participants who had learned a golf-putting task. The Reinvestment Scale contains 20 items drawn from three scales that were considered likely to predict individual propensity for reinvestment of controlled processing, with 12 items drawn from the SCS. Subsequent validation work indicated strong positive correlations between Reinvestment scores and "stress failure ratings" in varsity tennis $(n=12)$ and squash $(n=12)$ players (Masters et al., 1993). A recent study, again using a novice-learning paradigm in golf putting, similarly indicated that high reinvesters were more prone to "choking" (Poolton, Maxwell, \& Masters, 2004). Furthermore, structural equation modeling revealed a directional association from the accumulation of explicit knowledge to Reinvestment score to performance under anxiety.

\section{Current Experiments \??}

There is a growing body of literature documenting the negative impact of explicit monitoring on skilled performance. In addition, there is research examining the extent to which dispositional reinvestment or self-consciousness can predict skill failure under pressure. Thus far, these factors have been studied in isolation. In the present experiments, we aimed to replicate the results of Beilock, Carr, et al. (2002) regarding performance under single-task, dual-task, and skill-focused conditions, while extending their work by examining the moderating effects of pressure and dispositional reinvestment. In a second experiment we introduced a task-relevant dual-task condition that required participants to focus on a process goal, that is, the behavior the performer will engage in during performance (Kingston \& Hardy, 1997). 


\section{4 / Jackson, Ashford, and Norsworthy}

Researchers have previously highlighted several benefits associated with setting process goals such as increased confidence; however, the apparent paradox between the negative effect of explicit monitoring or conscious processing and the positive effect of focusing on process goals has also been noted (Mullen \& Hardy, 2000). Kingston and Hardy suggested that the same process goal could serve different functions depending on the skill level of the performer. This gives rise to the possibility that the function of process goals may change according to situational (e.g., perceived pressure) and dispositional (e.g., propensity for reinvestment) factors. In both experiments, therefore, we sought to enhance understanding of how attentional conditions interact with situational pressure and dispositional reinvestment to influence skilled performance.

\section{Experiment 1}

In Experiment 1, experienced field hockey players performed a dribbling task under single-task, skill-focused, or dual-task attention conditions, under both lowand high-pressure situations. In line with previous research on explicit monitoring, we predicted that skill-focused attention would disrupt performance relative to the single-task condition under low pressure. Reasoning that skill-focused attention engenders the same attentional state responsible for skill failure under pressure, we further predicted that performance under skill-focus conditions would be equivalent under low- and high-pressure conditions. In line with recent findings by Beilock, Carr, et al. (2002) and Beilock, Wierenga, and Carr (2002), we predicted that dualtask conditions would have a slight facilitative effect on performance under low pressure situations. Reasoning that performing a dual-task should prevent individuals from explicitly monitoring their performance, we further predicted that performance in the high-pressure dual-task condition would be better than performance in the high-pressure single-task condition (cf. Lewis \& Linder, 1997). Regarding the Reinvestment Scale, in accordance with the rationale for development of the scale, high reinvesters were predicted to be more susceptible to poor performance under pressure.

\section{Method}

\section{Participants and Measures}

Thirty-four field hockey players gave written consent to participate in the study. The sample comprised 15 males (mean age 22.8 years, $S D=2.7$ ) and 19 females (mean age 22.2 years, $S D=4.1$ ) who had competed at club ( $n=24)$, county $(n=6)$, or regional $(n=5)$ standard, and who were currently competing in fieldhockey leagues in the South of England. Participants had competed at their present level for a mean of 4.84 years $(S D=3.33)$.

Reinvestment Scale. The Reinvestment Scale contains 20 items drawn from three scales that were considered likely to predict individual propensity for reinvestment of controlled processing (Masters et al., 1993). Twelve items are taken from the Self-Consciousness Scale (e.g., "I'm aware of the way my mind works when I work through a problem"; Fenigstein et al., 1975). A further seven items are taken from the Rehearsal factor of the Emotional Control Questionnaire (e.g., "I often find myself thinking over and over about things that have made me 
angry"; Roger \& Nesshoever, 1987). The final item is taken from the Cognitive Failures Questionnaire ("Do you have trouble making up your mind?"; Broadbent, Cooper, Fitzgerald, \& Parkes, 1982). Data from Masters et al. (1993) indicated that the Reinvestment Scale has adequate internal reliability (Cronbach alpha $=0.86$ ) and test-retest reliability over a 4-month period $(r=.74)$.

In the present studies, each item was rated on a 5-point scale from 0 (extremely uncharacteristic) to 4 (extremely characteristic), resulting in a possible range of scores from 0 to 80. This scale is the same as that in the Self-Consciousness Scale, and was used to facilitate comparison of data from two of the Reinvestment subscale scores and equivalent normative subscale data from the Self-Consciousness Scale. In addition, a review of rating scales by Oppenheim (1992) revealed that 5-point scales yield more precise information about respondents' degree of agreement with each item, while Visser, Krosnick, and Lavrakas (2000) reported additional advantages for 5-point scales in terms of reliability and validity. To facilitate rating using the 5-point scale, the item from the Cognitive Failures Questionnaire was written in statement form: "I have trouble making up my mind." All other items remained the same.

State Anxiety. The cognitive and somatic anxiety subscales of the revised CSAI-2 (CSAI-2R; Cox, Martens, \& Russell, 2003) were used to assess state anxiety. Participants rated anxiety intensity on a 4-point scale anchored by 1 (not at all) and 4 (very much so) and anxiety direction on a 7-point scale from -3 (very debilitative) to +3 (very facilitative). Cox et al. (2003) reported acceptable internal reliability coefficients of .83 and .88 for cognitive and somatic anxiety subscales, respectively. In addition to the CSAI- $2 \mathrm{R}$, participants rated how much pressure they felt they had been under using a 7-point Likert scale from 1 (no pressure) to 7 (extreme pressure).

\section{Task and Procedure}

Individuals performed a field-hockey dribbling task on an indoor carpeted surface. The task required participants to use the "Indian dribble"1 to move the ball around a U-shaped slalom course using either their own stick or one provided by the experimenter. The course consisted of 10 cones spaced at 1-m intervals, with $0.5 \mathrm{~m}$ between the start gate and the first cone and between the last cone and the finish gate, creating a $10-\mathrm{m}$ course. Newtest PowerTimer 1.0 photoelectric cells were placed in the start and finish gates to record time to the nearest millisecond.

Upon entering the lab, participants completed a questionnaire eliciting background information, followed by the Reinvestment Scale. They were informed that they would complete 6 sets of 5 trials, that each trial would be timed, and that they would receive specific instructions before each set of trials. Finally, participants were told that if a significant error occurred in dribbling performance, such as missing a cone or using the wrong side of the hockey stick, the trial would be repeated (Beilock, Carr, et al., 2002). They were then given the opportunity to practice the task. After the relevant instructions but before the first of the 15

${ }^{1}$ The "Indian dribble" or "zigzag" refers to an advanced dribbling technique used to move the ball from left to right in front of the body. By rolling the left wrist to turn the stick over the ball, one uses the open side of the stick to control the ball (Anders \& Myers, 1999). 


\section{6 / Jackson, Ashford, and Norsworthy}

low-pressure and 15 high-pressure trials, participants completed the relevant items from the CSAI-2R. After the last of the low- and high-pressure trials, participants completed the pressure-rating item. The order of trials was counterbalanced both in terms of pressure and attention condition. Specifically, half of the participants performed the high-pressure trials before the low-pressure trials while the other half performed the low-pressure trials before the high-pressure trials. Within the blocks of low- and high-pressure trials, the order of attention conditions was counterbalanced with the order of presentation consistent across the two levels of pressure.

Single-Task. Participants were instructed to complete the trials as quickly and accurately as possible and were informed that the time taken to complete each trial would be used as the measure of performance.

Skill-Focus. In addition to the single-task condition instructions, participants were instructed to attend to the movement of their hands throughout the trial, so they could verbally indicate (by saying "up" or "down") whether their left hand was in a supine or prone position each time they heard a tone that sounded on a 6-second variable-interval schedule.

Dual-Task. The dual-task condition used by Beilock, Carr, et al. (2002) was changed slightly to improve consistency with the demands of the skill-focus condition, while attempting to minimize explicit monitoring. Specifically, in addition to the single-task condition instructions, participants were instructed to generate a random letter of the alphabet each time they heard a tone that sounded on the same variable-interval schedule used in the skill-focus condition. Random letter generation primarily places demands on the central executive component of working memory (Baddeley, 1986) and has been shown to minimize generation and use of explicit rules in motor tasks (MacMahon \& Masters, 2002). After each set of trials, participants were given a rest period of approximately 30 seconds during which they were required to count backward from 200 in 12 seconds to prevent them from reflecting on either their performance or the attention manipulation (Beilock, Carr, et al., 2002).

Pressure Manipulation. Pressure was induced by the presence of a confederate of the same gender as the participant who filmed the trials using a video camera mounted on a tripod. A cover story was also presented in which participants were informed that the footage would be used in a film about research into the basic skills of field hockey that the national governing body for field hockey was making for nationwide distribution. Both the presence of a video camera and evaluation apprehension have previously been used to manipulate pressure (e.g., Baumeister, 1984; Beilock \& Carr, 2001; Liao \& Masters, 2002; Masters, 1992; Mullen \& Hardy, 2000). At the end of the 30 experimental trials, participants completed the postexperiment questionnaire and were fully debriefed as to the nature and purpose of the study.

\section{Data Analysis}

Data screening using the Mahalanobis distance method identified one multivariate outlier, who was removed from the data set. A median split was then performed on the Reinvestment Scale score variable to create a group of high $(n=$ $14)$ and low reinvesters $(n=14)$, with mean scores of $52.50(S D=3.20$, range from 47 to 57 ) and 38.07 ( $S D=3.99$, range from 31 to 43 ), respectively. Participants with 
the median score were not included in subsequent analyses. Mean trial completion time served as the dependent variable and alpha was set at .05 but was adjusted using the Bonferroni correction where appropriate. To avoid issues regarding sphericity, and maintain consistency in reporting of results, output from multivariate tests using Wilks' Lambda are reported. Effect size is indicated by multivariate partial Eta squared $\left(\eta_{\mathrm{p}}{ }^{2}\right)$ in analyses of variance, and $95 \%$ confidence intervals (95\% CI) are reported for $t$-tests.

\section{Results of Experiment 1}

\section{Reinvestment and Performance}

Mean trial completion times for all participants are illustrated in Figure 1, where it can be seen, first, that times were slower under high pressure. Second, performance in the dual-task condition was faster than in the single-task condition, which in turn was faster than in the skill-focus condition. The data were entered into a $3 \times 2 \times 2$ (Attention Condition $\times$ Pressure $\times$ Reinvestment Group) ANOVA, with repeated measures on the first two factors. Results revealed significant main effects for Attention Condition, $F(2,25)=31.82, p<.001, \eta_{\mathrm{p}}{ }^{2}=.72$, and Pressure, $F(1,26)=36.49, p<.001, \eta_{\mathrm{p}}^{2}=.58$, but not Reinvestment Group, $F(1,26)=0.09$, $p=.76, \eta_{\mathrm{p}}{ }^{2}=.00$. A significant Attention Condition $\times$ Pressure interaction was also found, $F(2,25)=3.50, p<.05, \eta_{\mathrm{p}}{ }^{2}=.22$; however, the above were superseded by a significant three-way interaction between Attention Condition, Pressure, and Reinvestment Group, $F(2,25)=4.16, p<.05, \eta_{\mathrm{p}}{ }^{2}=.25$.

\section{Low Pressure $\square$ High Pressure}

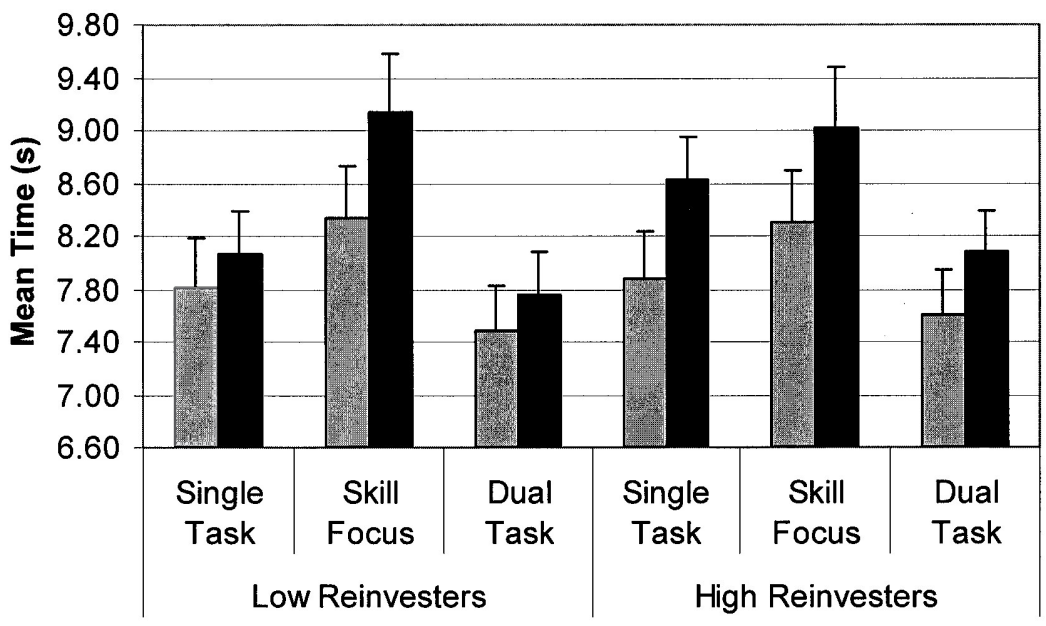

Figure 1 - Mean trial completion time (Experiment 1) for high and low reinvesters in the three attention conditions under low and high pressure, with standard error bars. 
To follow up the three-way interaction, we conducted separate $2 \times 2$ (Pressure $\times$ Reinvestment Group) ANOVAs for each attention condition. In the single-task condition, this revealed a significant interaction between Pressure and Reinvestment Group, $F(1,26)=5.46, p<.05, \eta_{\mathrm{p}}{ }^{2}=.17$, caused by the negative impact of pressure being greater in high reinvesters than in low reinvesters (difference: $M$ $=0.50 \mathrm{~s}, t[26]=2.34, p<.05,95 \%$ CI for difference: 0.06 to $0.93 \mathrm{~s}$ ). In the skillfocus and dual-task conditions, the Pressure $\times$ Reinvestment Group interaction was nonsignificant: skill focus, $p=.80$; dual-task, $p=.33$; as was the main effect for Reinvestment Group: skill focus, $p=.90$; dual-task, $p=.64$. In both cases there was a significant effect of Pressure: skill focus, $F(1,26)=27.91, p<.001, \eta_{\mathrm{p}}{ }^{2}=$ .52 ; dual-task, $F(1,26)=15.57, p<.01, \eta_{\mathrm{p}}{ }^{2}=.37$.

\section{Attention Conditions and Performance}

Contrasts between performance in the three attention conditions under low pressure, collapsed across Reinvestment Group, revealed a significant effect of Attention Condition, $F(2,31)=23.47, p<.001, \eta_{\mathrm{p}}{ }^{2}=.60$. Pairwise comparisons indicated that performance was significantly slower in the skill-focus than in the single-task condition $(M$ difference $=0.44 \mathrm{~s}, t[32]=5.41, p<.001,95 \% \mathrm{CI}$ for difference: 0.27 to $0.61 \mathrm{~s}$ ) and significantly faster in the dual-task than in the singletask condition $(M$ difference $=0.24 \mathrm{~s}, t[32]=3.68, p<.005,95 \% \mathrm{CI}$ for difference: 0.11 to $0.37 \mathrm{~s}$ ). A significant effect of Attention Condition was also evident in the high pressure trials, $F(2,31)=29.90, p<.001, \eta_{\mathrm{p}}{ }^{2}=.66$. Relative to single-task conditions, pairwise comparisons again indicated slower performance under skillfocus $(M$ difference $=0.66 \mathrm{~s}, t[32]=4.51, p<.001,95 \% \mathrm{CI}$ for difference: 0.36 to $0.95 \mathrm{~s})$ and faster performance under dual-task conditions $(M$ difference $=0.41 \mathrm{~s}$, $t[32]=6.71, p<.001,95 \% \mathrm{CI}$ for difference: 0.29 to $0.53 \mathrm{~s})$.

To test the specific prediction regarding performance under pressure in the dual-task and single-task conditions, we conducted a paired-samples $t$-test. This revealed that performance under pressure in the dual-task condition was significantly better than performance under pressure in the single-task condition, $t(32)$ $=6.71, p<.001$.

\section{Manipulation Checks}

Anxiety Intensity and Direction. Data from the cognitive and somatic anxiety subscales of the CSAI-2R were entered into a $2 \times 2$ (Pressure $\times$ Reinvestment Group) MANOVA with repeated measures on the Pressure factor. Results of the multivariate analyses revealed a significant effect of Pressure, Wilks' Lambda $=$ $0.29, F(4,23)=14.23, p<.001, \eta_{\mathrm{p}}{ }^{2}=.71$, and nonsignificant effects for both Reinvestment Group and the Pressure $\times$ Reinvestment Group interaction. Univariate analyses of the Pressure effect revealed significant differences for all four factors: Cognitive Intensity: $F(1,26)=20.29, p<.001, \eta_{\mathrm{p}}{ }^{2}=.44$; Cognitive Direction: $F(1$, $26)=32.45, p<.001, \eta_{\mathrm{p}}{ }^{2}=.56$; Somatic Intensity: $F(1,26)=23.75, p<.001, \eta_{\mathrm{p}}{ }^{2}$ $=.48$; Somatic Direction: $F(1,26)=9.71, p<.01, \eta_{\mathrm{p}}{ }^{2}=.27$. The changes from low pressure to high pressure were in the expected direction for Cognitive Intensity (17.14 to 22.00), Cognitive Direction (2.68 to -1.25), and Somatic Intensity (13.78 to 17.14). Unexpectedly, increased somatic anxiety intensity was perceived as being more facilitative (Somatic Direction: 2.68 to 5.86), the reason for which is unclear and may be a spurious result. 
Pressure Ratings. The pressure-rating question revealed greater perceived pressure in the high-pressure $(M=4.57, S D=1.48)$ than in the low-pressure trials $(M=2.93, S D=1.27)$. A $2 \times 2$ (Pressure $\times$ Reinvestment Group) ANOVA with repeated measures on the Pressure factor revealed a significant main effect for Pressure, $F(1,26)=54.36, p<.001, \eta_{\mathrm{p}}^{2}=.68$, and a nonsignificant Pressure $\times$ Reinvestment Group interaction, $p=.21$, indicating equivalent increases in perceived pressure for low and high reinvesters.

Dual-Task Performance. In total, only 11 errors were made in verbalizing hand position and these did not differ between reinvestment groups, $t(26)=0.30$, $p=.77$. There were no errors or omissions in the random letter-generation task. Inspection of the videotapes revealed that nine of the errors in the skill-focused condition were attributable to the transitional position of the hands when the tone sounded.

\section{Discussion, Experiment 1}

Results from the different attention conditions were consistent with the findings of Beilock, Carr, et al. (2002). In particular, the finding that low-pressure performance was fastest under dual-task conditions and slowest when participants monitored hand position replicated Beilock, Carr, et al.'s results for skilled soccer players and experienced golfers using the auditory word-monitoring and tone-monitoring task, respectively. Specifically, Beilock, Carr, et al. found that novices dribbling soccer balls using their dominant foot were slower under dual-task conditions than under skill-focused conditions whereas the reverse was true for experienced players. It should be noted that random letter generation has previously been found to suppress primary motor task performance (Bright \& Freedman, 1998; Masters, 1992; MacMahon \& Masters, 2002); however, these studies required participants to generate letters every 1.5 seconds, increasing to every 1.0 seconds to maintain task difficulty (Baddeley, 1966).

The dual-task condition in the present study involved auditory monitoring for a tone, followed by generation of a random letter. With the comparatively low frequency of letter generation (every $6.0 \mathrm{~s}$ ), it would seem likely that the attentional demands were more akin to tone monitoring. In addition, research showing suppressed motor performance under random letter-generation conditions has used novice performers for whom concurrent subvocal speech and explicit attention to rules may play a supportive role (Kimble \& Perlmuter, 1970). Consequently, a secondary task that suppresses this process would be expected to have a detrimental effect on performance. By contrast, expert performance requires little conscious online attention so that attending to secondary tasks should leave performance on the primary motor task unaffected (Beilock, Carr, et al., 2002).

What is less clear is why performance should be better under dual-task than single-task conditions. This finding occurred in Beilock, Carr, et al.'s study of soccer players, and Beilock, Wierenga, et al. (2002) also reported a marginally significant improvement in experienced golfers' putting performance when using a concurrent secondary word-monitoring task. One possibility is that the external focus required to monitor auditory tones attenuated any residual explicit monitoring that was present under single-task conditions. The benefits of an external focus have been illustrated in both learning (e.g., Wulf, Lauterbach, \& Toole, 1999) and performance (Singer, Lidor, \& Caraugh, 1993) contexts, and Masters and Maxwell 
(2004) recently highlighted the similarities between reinvestment / explicit monitoring accounts of skill failure and those framed in terms of performers adopting an internal focus of attention.

In terms of the effect of pressure, data in the single-task condition were consistent with Masters et al.'s (1993) prediction that high reinvesters would be more susceptible to choking under pressure: high reinvesters slowed significantly more than low reinvesters in the high-pressure trials. However, the finding that performance deterioration in the skill-focus condition was compounded by high pressure is inconsistent with the skill-focus condition, replicating the effect of pressure. One possibility is that performers can vary the extent to which they explicitly monitor performance and that this results in varying degrees of performance disruption. Thus, rather than explicit monitoring being a single discrete state, there may be varying degrees to which performers explicitly monitor their performance. Accordingly, skill-focused and pressure-induced explicit monitoring may have additive effects, resulting in even poorer performance when both occur simultaneously. This interpretation is consistent with recent challenges to the dichotomous account of explicit and implicit processes, whereby researchers have argued that learning invariably involves a contribution from both processes (see Jackson \& Farrow, 2005).

Consistent with this view, Smeeton, Williams, Hodges, and Ward (2005) found that the degree to which decision time slowed under pressure in a group who had learned a skill explicitly was strongly and linearly related $(r=.76)$ to the number of explicit rules they recalled at the end of the experiment. This would also help account for the finding that performance under dual-task conditions was still poorer under high pressure, albeit performance returned to a level approximately equivalent to the low-pressure single-task condition. Thus, the dual-task condition can be viewed as counteracting the tendency for pressure to invoke explicit monitoring.

\section{Experiment 2}

In Experiment 1, adopting a skill focus had a detrimental effect on skilled motor performance while the dual-task condition facilitated performance. In Experiment 2 we sought to replicate the first finding while examining the effects of focusing on a task-relevant secondary task: process goals. Process goals refer to behaviors the performer will engage in during performance (Kingston \& Hardy, 1997, p. 279), and Kingston and Hardy gave an example of a highly successful pistol shooter who described using a mental checklist of words representing "every single step involved in shooting a shot" to maintain motivation for quality training (p. 280). Although somewhat limited, researchers have previously highlighted several benefits associated with setting process goals. For example, Kingston and Hardy found that club golfers who set process goals over a 1-year period experienced greater increases in self-efficacy and concentration, and decreases in cognitive anxiety, than a group setting performance goals. In addition, Filby, Maynard, and Graydon (1999) found that performers who used a combination of outcome and process goals scored significantly more points on a kicking accuracy task than those setting either process or outcome goals alone.

When set in the context of explicit monitoring theories, process goals present an apparent paradox in that they appear to encourage performers to focus consciously on aspects of performance that are normally performed automatically (Hardy et al., 
1996). Kingston and Hardy (1997) proposed one explanation, namely that process goals may serve different functions in skilled and less-able performers. For skilled performers, Kingston and Hardy argued that process goals function as holistic, conceptual cues for the to-be-performed behavior. For less-able performers, they suggested that process goals enhance performance through focusing attention on key elements of performance. This functional account is consistent with the finding that explicit monitoring facilitates performance in novices but disrupts skilled performance (Beilock, Carr, et al., 2002). In the context of reinvestment theory and performance under pressure, it gives rise to the possibility that process goals may invoke different attentional functions according to changes in perceived pressure and/or individual propensity for exerting conscious control over performance.

In Experiment 2 we sought to examine this issue. Specifically, we predicted that process goals would function as conceptual cues under low pressure, but would trigger explicit monitoring under high pressure. We therefore hypothesized that focusing on a process goal would facilitate performance under low pressure but would have a negative impact on performance under high pressure. Consistent with conceptualization of the Reinvestment Scale, we further predicted that this effect would be most pronounced in high reinvesters.

\section{Method}

\section{Participants and Measures}

Twenty-five male varsity soccer players (mean age 20.4 years, $S D=1.1$ ) consented in writing to take part in the study. Participants were current representatives of university first or second teams and had played competitive-level soccer for a mean of 12.44 years $(S D=2.71)$. Twenty-one participants were right-footed and four were left-footed; all played in outfield positions.

The Reinvestment Scale and the cognitive and somatic items from the CSAI2R were again used (see Experiment 1). As in Experiment 1, a single question assessing perceived pressure was used as an additional manipulation check.

\section{Design and Procedure}

The soccer-dribbling task used by Beilock, Carr, et al. (2002) was chosen for the present study. The task was set up on a carpeted surface and required participants to dribble a soccer ball as quickly and accurately as possible through a series of 6 cones spaced at 1.5 -m intervals (total length $=10.5 \mathrm{~m}$ ) using the instep and outstep of their dominant foot. Newtest PowerTimer 1.0 photoelectric cells were again placed in the start and finish gates to record trial completion time to the nearest millisecond. The procedure for Experiment 2 was the same as for Experiment 1 with the exception that participants completed 3 trials in each combination of attention and pressure, generating a total of 18 trials. Again, trials were counterbalanced with respect to both attention and pressure conditions.

Single-Task. Participants were instructed to complete the task as quickly and accurately as possible and were informed that the time taken to complete each trial would be used as the measure of performance.

Skill-Focus. Participants were additionally instructed to attend to the side of their foot that made contact with the ball throughout the trial. To ensure adher- 


\section{2 / Jackson, Ashford, and Norsworthy}

ence to this instruction, participants were instructed, upon hearing a tone, to report which side of their foot had just touched the ball. The tone sounded on a 6-second variable interval schedule.

Process Goal. In the process goal condition, participants were asked to set a single goal regarding their ball-dribbling behavior to try to maximize success on the task. To help them formulate their goal, the experimenter gave the example of a golfer ensuring that his/her alignment was appropriate prior to playing a shot (Kingston \& Swain, 1999). Participants then restated their process goal prior to beginning each trial in the process goal condition.

Pressure Manipulation. As in Experiment 1, a video camera and cover story were used to manipulate pressure. Specifically, prior to the high pressure trials the participants were informed that they would be filmed and that the footage would be used by coaches at a local English Premiership team's soccer academy to evaluate their performance relative to academy players of different ages. At the end of the study, the participants were fully debriefed as to the nature and purpose of the study.

\section{Results of Experiment 2}

A median split was performed on total Reinvestment Scale score to create a group of high $(n=13)$ and low reinvesters $(n=12)$, with mean scores of $52.42(S D=$ 5.65 , range from 46 to 64 ) and 38.00 ( $S D=6.03$, range from 28 to 44$)$, respectively. Mean trial completion time served as the dependent variable and alpha was set at .05 . Multivariate results are reported, and effect size is indicated by multivariate partial Eta squared $\left(\eta_{\mathrm{p}}{ }^{2}\right)$.

\section{Attention, Reinvestment, and Performance}

The analysis was focused on two questions. First, how did setting a process goal affect performance relative to the single-task and skill-focus conditions? Second, did performance in the process goal condition interact with pressure and/ or reinvestment group? To answer these questions, we first entered the data into a $3 \times 2 \times 2$ (Attention Condition $\times$ Pressure $\times$ Reinvestment Group) ANOVA, with repeated measures on the first two factors. Results of the multivariate analysis revealed a significant main effect for Attention Condition, $F(2,22)=38.65, p<$ $.001, \eta_{\mathrm{p}}{ }^{2}=.78$, with planned contrasts indicating faster performance in the singletask condition $(M=5.38, S E=1.07)$ than in the process goal condition $(M=5.79$, $S E=1.24), F(1,23)=14.00, p<.001, \eta_{\mathrm{p}}{ }^{2}=.38$, which in turn was faster than the skill-focus condition $(M=6.20, S E=1.11), F(1,23)=16.52, p<.001, \eta_{\mathrm{p}}{ }^{2}=.42$. The hypothesis that process goals would facilitate performance under low pressure but have an adverse effect on performance under high pressure was not supported. Specifically, the interaction between Attention Condition and Pressure, $F(2,46)=$ $0.36, p=.70, \eta_{\mathrm{p}}{ }^{2}=.02$, and its higher-order interaction with Reinvestment Group, $F(2,46)=0.13, p=.88, \eta_{\mathrm{p}}{ }^{2}=.01$, were nonsignificant. Thus, the adverse effect of attending to a process goal was equivalent under low- and high-pressure conditions for both low and high reinvesters.

The main effect of Attention Condition was moderated by a significant Attention Condition $\times$ Reinvestment Group interaction, $F(2,22)=4.27, p<.05$, 


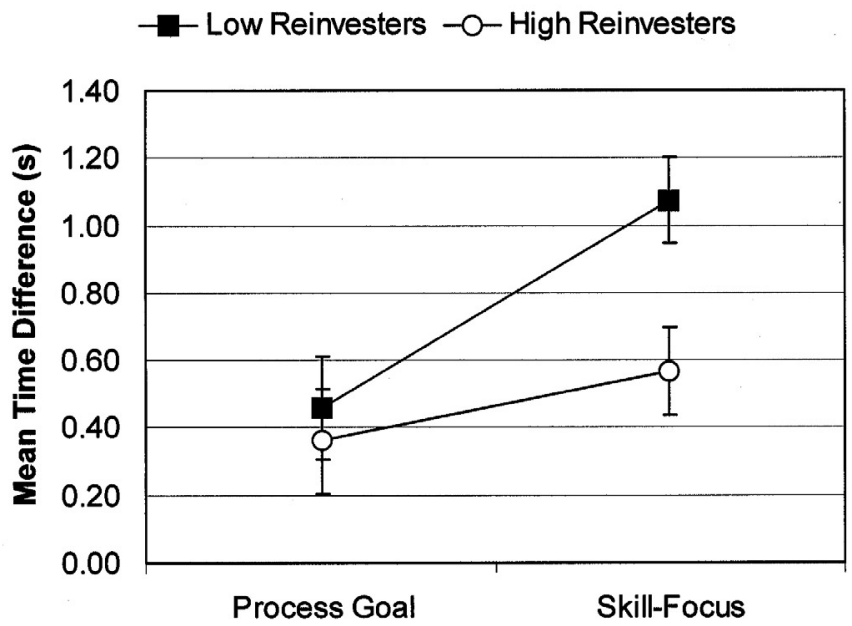

Figure 2-Mean trial completion time differences relative to the single-task condition (Experiment 2) for high and low reinvesters, with standard error bars.

$\eta_{\mathrm{p}}{ }^{2}=.28$. Follow-up analysis revealed similar times for high and low reinvesters in the process goal condition $(M$ Difference $=0.09 \mathrm{~s} ; t[23]=0.42, p=.68 ; 95 \% \mathrm{CI}$ for Difference $=-0.36$ to 0.55$)$ and significantly longer times for low reinvesters than for high reinvesters in the skill-focus condition ( $M$ Difference $=0.50 \mathrm{~s} ; t[23]$ $=2.75, p<.05 ; 95 \% \mathrm{CI}$ for Difference $=0.13$ to 0.88 ) relative to the single-task condition (see Figure 2). In terms of the effect of pressure, planned comparisons between performance under low and high pressure in the single-task condition revealed a marginally significant difference in the expected direction for high reinvesters $(M$ Difference $=0.25 \mathrm{~s} ; t[11]=1.75, p=.05)$ and a null finding for low reinvesters $(M$ Difference $=0.00 \mathrm{~s} ; t[12]=0.05, p=.96)$. However, the main effect and higher-order interactions associated with Pressure were nonsignificant in the three-way ANOVA.

Given the detrimental effect of the process goal condition, we decided to conduct an a posteriori analysis of the nature of process goals that were set. Two types of process goal were identified: movement related goals $(n=16)$ which included reference to a specific technique or movement (e.g., "keep loose with knees bent"), and position related goals $(n=8)$ that specified either the position of the ball in relation to the performer or the performer in relation to the course (e.g., "keep over the ball with my body"). All but one of the participants set a goal that could be classified as movement related or position related. Classification of the goals by an independent rater who was given the goal statements and definitions revealed $100 \%$ agreement with the experimenter's classification. A $2 \times 2$ (Attention Condition $\times$ Goal Type) ANOVA was subsequently performed that revealed a significant interaction, $F(1,22)=18.92, p<.001, \eta_{\mathrm{p}}{ }^{2}=.46$. Movement related goals, but not position related ones, were found to have a detrimental effect on performance relative to the single-task condition (see Figure 3). 


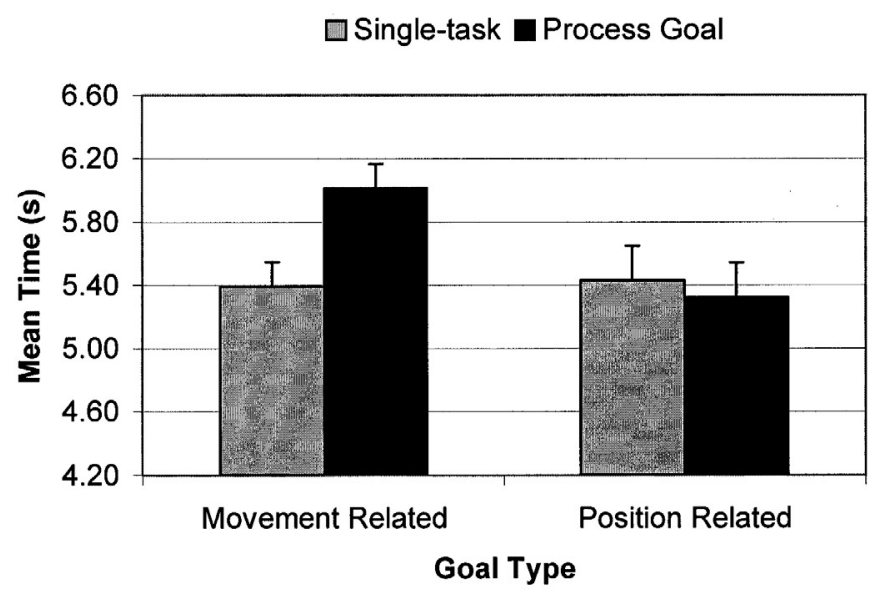

Figure 3 - Mean trial completion times for participants setting movement related (n $=16)$ and position related $(n=8)$ process goals, with standard error bars.

\section{Manipulation Checks}

Anxiety Intensity and Direction. Data from the cognitive and somatic anxiety subscales of the CSAI-2R revealed changes in the expected direction for all variables from the low-pressure to high-pressure condition: Cognitive Intensity: 16.49 to 19.60; Cognitive Direction: 0.51 to -4.30 ; Somatic Intensity: 14.15 to 16.89 ; Somatic Direction: 2.86 to 0.16 . Results of a $2 \times 2$ (Pressure $\times$ Reinvestment Group) MANOVA with repeated measures on the Pressure factor revealed a significant effect of Pressure, $F(4,20)=9.05, p<.001, \eta_{\mathrm{p}}{ }^{2}=.64$, and nonsignificant effects for both Reinvestment Group and the Pressure $\times$ Reinvestment Group interaction ( $p=.44$ and $p=.19$, respectively). Results of the univariate analysis for Pressure revealed significant differences in all four factors: Cognitive Intensity: $F(1,23)=$ $32.53, p<.001, \eta_{\mathrm{p}}^{2}=.59$; Cognitive Direction: $F(1,23)=14.25, p<.01, \eta_{\mathrm{p}}{ }^{2}=.38$; Somatic Intensity: $F(1,23)=15.67, p<.01, \eta_{\mathrm{p}}{ }^{2}=.41$; Somatic Direction: $F(1,23)$ $=6.03, p<.05, \eta_{\mathrm{p}}^{2}=.21$.

Pressure Ratings. Data from the pressure rating scale revealed significantly higher perceived pressure in the high-pressure trials $(M=4.84)$ than in the lowpressure trials $(M=3.19), p<.001, \eta_{\mathrm{p}}^{2}=.76$, a difference that was equivalent for low and high reinvesters (Pressure $\times$ Reinvestment Group interaction: $p=.59$ ).

Dual-Task Performance. Participants made a total of only three errors when verbalizing which side of the foot had just touched the ball.

\section{Discussion, Experiment 2}

The hypothesis that process goals would have differential effects on performance according to dispositional reinvestment and pressure was not supported. 
Movement related process goals were found to be detrimental to performance regardless of dispositional reinvestment or situational pressure. This suggests a need to distinguish between different types of process goal that may be associated with different attentional functions. On the one hand, process goals relating to skilled movements may encourage explicit monitoring while those relating to more strategic features of positioning may direct the performer's attention away from the physical movements being performed, functioning as holistic cues for the required behavior (Kingston \& Hardy, 1997; see also Jackson \& Willson, 1999). Consistent with this point, Kingston and Hardy identified "a process goal orientation as the most appropriate primary focus for competition" (p. 290) but acknowledged the paradoxical nature of advice to set process goals yet not engage in conscious processing.

This suggestion is also consistent with Beilock, Carr, et al.'s (2002) proposed distinction between self-regulatory attention and skill-focused attention. Specifically, process goals relating to strategic features of a task may engage attentional processes that serve higher level, metacognitive roles, thereby preventing focus on the stepby-step processes governing skill execution.

Consistent with Experiment 1, skill-focused attention again had a detrimental effect on dribbling speed relative to the single-task condition, and high reinvesters again showed a greater, albeit marginally significant, tendency toward poorer performance under pressure in the single-task condition. Interestingly, the adverse effect of adopting a skill focus was more pronounced in low reinvesters. This is consistent with an extension of the "acclimatization" hypothesis (Baumeister, 1984) which suggests that low reinvesters may be less used to focusing on the processes governing performance, hence being more prone to choking. Although apparently at odds with one another, the reinvestment and acclimatization accounts are not entirely irreconcilable. If low reinvesters tend to engage in explicit monitoring less than high reinvesters, then instructing low reinvesters to do so would be predicted to have a more negative impact on performance than for high reinvesters. At the same time, low reinvesters might still have a lower propensity for actually engaging in this process under pressure. Before speculating further, there is a need for additional research to establish whether this finding is robust, particularly given that the equivalent effect in Experiment 1 was not statistically significant.

In terms of pressure, it is unclear why the same manipulation had a less negative impact on performance than in Experiment 1, despite resulting in comparable changes in preperformance anxiety intensity and pressure ratings. One possibility relates to task complexity. The hockey-dribbling task may be considered more complex than the soccer-dribbling task by virtue of the U-shaped as opposed to "straight line" design of the course and the need to use a hand-held implement to control a smaller ball. This may have decreased the sensitivity of the soccer-dribbling task to performance effects resulting from changes in situational pressure. For example, MacMahon and Masters (2002) found that random letter generation did not disrupt performance on short $(1.5 \mathrm{~m})$ straight golf putts, but that it significantly suppressed performance on longer $(3.0 \mathrm{~m})$, curved putts. Thus, although the pattern of results with respect to pressure was largely consistent with Experiment 1 , a degree of caution should be expressed when interpreting nonsignificant effects relating to pressure. 


\section{General Discussion}

The aim of the present studies was to evaluate the relationship between attentional focus, dispositional reinvestment, and skill failure under pressure. Researchers have proposed that skill-focused attention, or explicit monitoring, is central to choking but have tended to manipulate attention or pressure in isolation, thereby providing only indirect evidence of the link between the two (Liao \& Masters, 2002). The present studies manipulated both attentional focus and pressure in addition to examining the relative performance of individuals with a low and high propensity for reinvesting conscious control.

The results were consistent with explicit monitoring theories of skill failure, with the cumulative detrimental effects of pressure and skill focus observed in Experiment 1 suggesting that explicit processes could be activated to varying degrees with resultant varying degrees of disruption. However, two possible limitations should be noted. First, a possible limitation of the skill-focus manipulation used in this and previous research (Beilock, Carr, et al., 2002) is that one cannot ensure that participants actually monitor their movements in accordance with the instruction. Specifically, although they are asked to monitor their movements, they are only asked to report one feature of their movement in response to the tone. In this situation, participants may attempt to recall hand or foot position each time they hear the tone but not actually monitor their movements over the duration of the trial. We argue that the low number of tones on each trial coupled with the very low number of judgment errors and negative impact on performance mitigates against this possibility, but it cannot entirely be discounted.

A second potential limitation is that the negative impact of skill-focus instructions and focusing on movement related goals may have been due to participants performing the skill in a novel fashion. Thus, being asked to monitor features of performance is likely to be novel for skilled performers and would also account for the differential effects of such instructions in skilled and novice participants observed in previous work (Beilock, Carr, et al., 2002). Following this line of reasoning, it is also possible that skilled soccer players would routinely monitor elements of position on this task. Consequently, setting an explicit position related goal could be accommodated within their normal focus of attention. Set against this possibility, performance under dual-task conditions might also be considered novel, particularly if performers are prevented from routinely monitoring elements of positioning. Nevertheless, additional research is needed to establish to what degree the breakdown in skilled performance under skill-focus conditions is due to explicit monitoring and to what extent it is due to performing the skill in a novel fashion.

One other possibility warrants mentioning, as it relates to the conceptual distinction between explicit monitoring and reinvestment of conscious control. While researchers have highlighted similarities between explicit monitoring and reinvestment accounts of skill failure (Masters \& Maxwell, 2004), the terms are logically distinct insofar as instructions to monitor and report a particular feature of performance encourage explicit monitoring but do not specifically encourage conscious control. Thus, it is possible that explicit monitoring has a general disruptive effect on motor performance and that additional disruption occurs when performers attempt to apply explicit rules to control as well as monitor their movements. 
This would potentially account for the additive detrimental effect of skill focus and pressure observed in Experiment 1 if the skill-focus instruction prompts explicit monitoring while increased pressure prompts the participant to attempt to control those elements that are being monitored. For example, in their initial validation of the Reinvestment Scale, Masters et al. (1993) found that performance on a rod-tracing task was considerably slower under pressure but that decrements in performance were equivalent for both low and high reinvesters. In explaining their results, Masters et al. appealed to the lack of explicit rules associated with the task and subsequently demonstrated interactions between reinvestment and choking on a more complex, rule-bound, golf-putting task.

The theoretical and practical significance of this distinction could be determined by employing dual-task methodology to assess "on-line" attentional demands. For example, Gray (2004) measured performers' ability to monitor extraneous and task-relevant information while performing a simulated baseball task under a variety of conditions. He found that experts were better able to report extraneous information (tone frequency) than novices, and that expert batters' performance over the previous 24 trials was strongly correlated $(r=.89)$ with their ability to make a retrospective judgment about whether the bat had been moving downward or upward at the instant a tone sounded. If explicit monitoring is necessary but not sufficient for reinvestment of conscious control, the latter should be more demanding of attention, as reflected in a reduced ability to attend to extraneous information during performance.

Similar techniques could be used to shed additional light on the processes underlying the behavioral effects observed in the present experiments. For example, the results of Experiment 2 highlighted differential performance effects associated with focusing on different types of process goals but did not establish a direct link between attending to movement related goals and explicit monitoring. If certain process goals cue explicit monitoring while others cue automatic execution of the skill, this should be reflected in the ability to monitor task-relevant and extraneous information. Similarly, the fact that the Reinvestment Scale takes its name from the process of reinvesting conscious control is perhaps a little misleading, as the scale does not attempt to measure the process of reinvestment directly but instead aims to bring together conceptually linked items that predict this process.

Consequently, while the results of the present experiments were largely consistent with the prediction that high reinvesters would show a greater propensity for choking (Masters et al., 1993), further research is needed to establish the validity of the scale in terms of predicting the process of reinvesting conscious control. Again, this could be achieved via on-line monitoring of attentional demands during task performance. Finally, there is a need to evaluate the predictive validity of the Reinvestment Scale in terms of choking and/or the process of reinvestment in an ecologically valid setting to supplement evidence obtained from novice performers in learning paradigms and that from subjective ratings of propensity for skill failure.

As Kimble and Perlmuter (1970) observed, "the act of paying attention to... performances or describing the steps as they occur tends to destroy the automaticity of such behavior" (p.375). A growing body of research is supportive of this statement and the present results provide further support for explicit monitoring theories of choking and predictions regarding the relative vulnerability of high reinvesters to 
such effects. At the same time, issues still remain regarding the nature and number of processes underlying skill failure, as well as the validity of the Reinvestment Scale in terms of predicting such processes.

\section{References}

Anders, M., \& Myers, S. (1999). Field hockey: Steps to success. Champaign, IL: Human Kinetics.

Baddeley, A.D. (1966). The capacity for generating information by randomization. Quarterly Journal of Experimental Psychology, 18, 119-129.

Baddeley, A.D. (1986). Working memory. Oxford: University Press.

Baumeister, R.F. (1984). Choking under pressure: Self-consciousness and paradoxical effects of incentives on skilful performance. Journal of Personality and Social Psychology, 46, 610-620.

Baumeister, R.F., \& Showers, C.J. (1986). A review of paradoxical performance effects: Choking under pressure in sports and mental tests. European Journal of Social Psychology, 16, 361-383.

Beilock, S.L., \& Carr, T.H. (2001). On the fragility of skilled performance: What governs choking under pressure? Journal of Experimental Psychology: General, 130, 701725 .

Beilock, S.L., Carr, T.H., MacMahon, C., \& Starkes, J.L. (2002). When paying attention becomes counterproductive: Impact of divided versus skill-focused attention on novice and experienced performance of sensorimotor skills. Journal of Experimental Psychology: Applied, 8, 6-16.

Beilock, S.L., Wierenga, S.A., \& Carr, T.H. (2002). Expertise, attention, and memory in sensorimotor skill execution: Impact of novel task constraints on dual-task performance and episodic memory. Quarterly Journal of Experimental Psychology, 55A, 1211-1240.

Bright, J.E.H., \& Freedman, O. (1998). Differences between implicit and explicit acquisition of a complex motor skill under pressure: An examination of some evidence. British Journal of Psychology, 89, 249-263.

Broadbent, D.E., Cooper, P.F., Fitzgerald, P., \& Parkes, K.R. (1982). The Cognitive Failures Questionnaire (CFQ) and its correlates. British Journal of Clinical Psychology, 21, $1-16$.

Carver, C.S., \& Scheier, M.F. (1978). Self-focusing effects of dispositional self-consciousness, mirror presence, and audience presence. Journal of Personality and Social Psychology, 36, 324-332.

Cox, R.H., Martens, M.P., \& Russell, W.D. (2003). Measuring anxiety in athletics: The Revised Competitive State Anxiety Inventory-2. Journal of Sport \& Exercise Psychology, 25, 519-533.

Fenigstein, A., \& Carver, C.S. (1978). Self-focusing effects of heartbeat feedback. Journal of Personality and Social Psychology, 36, 1241-1250.

Fenigstein, A., Scheier, M.F., \& Buss, A.H. (1975). Public and private self-consciousness: Assessment and theory. Journal of Consulting and Clinical Psychology, 43, 522527.

Filby, W.C.D., Maynard, I.W., \& Graydon, J.K. (1999). The effect of multiple-goal strategies on performance outcomes in training and competition. Journal of Applied Sport Psychology, 11, 230-246. 
Ford, P., Hodges, N.J., \& Williams, A.M. (2005). Online attentional-focus manipulations in a soccer-dribbling task: Implications for the proceduralization of motor skills. Journal of Motor Behavior, 37, 386-394.

Gray, R. (2004). Attending to the execution of a complex sensorimotor skill: Expertise differences, choking, and slumps. Journal of Experimental Psychology: Applied, 10, 42-54.

Hardy, L., Mullen, R., \& Jones, G. (1996). Knowledge and conscious control of motor actions under stress. British Journal of Psychology, 87, 621-636.

Jackson, R.C., \& Farrow, D. (2005). Implicit perceptual training: How, when, and why? Human Movement Science, 24, 308-325.

Jackson, R.C., \& Willson, R.J. (1999). Using 'swing thoughts' to prevent paradoxical performance effects in golf putting. In M.R. Farrally \& A.J. Cochran (Eds.), Science and golf III (pp. 166-173). Champaign, IL: Human Kinetics.

Kimble, G.A., \& Perlmuter, L.C. (1970). The problem of volition. Psychological Review, 77, 361-384.

Kingston, K., \& Hardy, L. (1997). Effects of different types of goals on processes that support performance. The Sport Psychologist, 11, 277-293.

Kingston, K., \& Swain, A. (1999). Goal orientations and state goals: Research in golf and implications for performance. In M.R. Farrally \& A.J. Cochran (Eds.), Science and golf III (pp. 150-157). Champaign, IL: Human Kinetics.

Lewis, B.P., \& Linder, D.E. (1997). Thinking about choking? Attentional processes and paradoxical performance. Personality and Social Psychology Bulletin, 23, 937-944.

Liao, C., \& Masters, R.S.W. (2001). Analogy learning: A means to implicit learning. Journal of Sports Sciences, 19, 307-319.

Liao, C., \& Masters, R.S.W. (2002). Self-focused attention and performance failure under psychological stress. Journal of Sport \& Exercise Psychology, 24, 289-305.

MacMahon, K.M.A., \& Masters, R.S.W. (2002). The effects of secondary tasks on implicit motor skill performance. International Journal of Sport Psychology, 33, 307-324.

Masters, R.S.W. (1992). Knowledge, knerves and know-how: The role of explicit versus implicit knowledge in the breakdown of a complex motor skill under pressure. British Journal of Psychology, 83, 343-358.

Masters, R.S.W., \& Maxwell, J.P. (2004). Implicit motor learning, reinvestment and movement disruption: What you don't know won't hurt you. In A.M. Williams \& N.J. Hodges (Eds.), Skill acquisition in sport: Research, theory and practice (pp. 207-228). London: Routledge.

Masters, R.S.W., Polman, R.C.J., \& Hammond, N.V. (1993). Reinvestment: A dimension of personality implicated in skill breakdown under pressure. Personality and Individual Differences, 14, 655-666.

Maxwell, J.P., Masters, R.S.W., \& Eves, F.F. (2000). Explicit versus implicit motor learning: Dissociating selective and unselective modes of skill acquisition via feedback manipulation. Journal of Sports Sciences, 18, 559.

Maxwell, J.P., Masters, R.S.W., Kerr, E., \& Weedon, E. (2001). The implicit benefit of learning without errors. The Quarterly Journal of Experimental Psychology, 54A, 1049-1068.

Mullen, R., \& Hardy, L. (2000). State anxiety and motor performance: Testing the conscious processing hypothesis. Journal of Sports Sciences, 18, 785-799.

Oppenheim, A.N. (1992). Questionnaire design, interviewing and attitude measurement (2nd ed.). New York: Continuum. 
Poolton, J., Maxwell, J., \& Masters, R. (2004). Rules for reinvestment. Perceptual and Motor Skills, 99, 771-774.

Roger, D., \& Nesshoever, W. (1987). The construction and preliminary validation of a scale for measuring emotional control. Personality and Individual Differences, 8 , 527-534.

Singer, R.N., Lidor, R., \& Cauraugh, J.H. (1993). To be aware or not aware? What to think about while learning and performing a motor skill. The Sport Psychologist, 7, 1930 .

Smeeton, N.J., Williams, A.M., Hodges, N.J., \& Ward, P. (2005). The relative effectiveness of various instructional approaches in developing anticipation skill. Journal of Experimental Psychology: Applied, 11, 98-110.

Visser, P.S., Krosnick, J.A., \& Lavrakas, P.J. (2000). Survey research. In H.T. Reiss \& C.M. Judd (Eds.), Handbook of research methods in social and personality psychology (pp. 223-252). Cambridge: University Press.

Wulf, G., Lauterbach, B., \& Toole, T. (1999). The learning advantages of an external focus of attention in golf. Research Quarterly for Exercise and Sport, 70, 120-126.

Manuscript submitted: December 29, 2004

Revision accepted: October 13, 2005 